\title{
Lidil
}

Revue de linguistique et de didactique des langues

46 | 2012

Typologie et description linguistiques

\section{Valence sémantique et diathèses en tibétain (Lhasa)}

Camille Simon

\section{OpenEdition}

Journals

Édition électronique

URL : http://journals.openedition.org/lidil/3248

DOI : $10.4000 /$ lidil.3248

ISSN : $1960-6052$

Éditeur

UGA Éditions/Université Grenoble Alpes

Édition imprimée

Date de publication : 15 décembre 2012

Pagination : 127-151

ISBN : $978-2-84310-236-3$

ISSN : $1146-6480$

Référence électronique

Camille Simon, «Valence sémantique et diathèses en tibétain (Lhasa) », Lidil [En ligne], 46 | 2012, mis en ligne le 15 juin 2014, consulté le 20 avril 2019. URL : http://journals.openedition.org/lidil/3248 :

DOI : $10.4000 /$ lidil.3248

(c) Lidil 


\title{
Valence sémantique et diathèses en tibétain (Lhasa)
}

\author{
Camille Simon*
}

\begin{abstract}
RÉSUMÉ
Les descriptions de la syntaxe du tibétain ont depuis longtemps mis en évidence plusieurs caractéristiques qui montrent que les questions de valence verbale dans cette langue ne peuvent être traitées à un niveau simplement syntaxique, mais doivent intégrer des composantes pragmatiques et sémantiques essentielles. Cet article vise donc à mettre en avant ces questions d'ordre sémantique, essentielles pour la compréhension de la notion de valence. Nous proposons tout d'abord une définition de la valence verbale en tibétain, puis une description de deux diathèses et enfin d'un autre exemple de variation du marquage morpho-syntaxique des actants dans l'énoncé.
\end{abstract}

\section{ABSTRACT}

Descriptions of the syntax of Tibetan language have long drawn attention to several characteristics suggesting that the issues of verb valency in this language cannot be processed at a purely syntactic level. Essential pragmatic and semantic features must be incorporated in the definition of valency. This article aims to underline these aspects of semantic features important to our understanding of this notion. First I will deliver a semantic definition of valency in the Tibetan language, and continue with a description of two diatheses and another type of variation of the case-marking of arguments.

\section{Introduction}

Les descriptions de la syntaxe du tibétain ont depuis longtemps mis en évidence plusieurs caractéristiques qui montrent que les questions de valence verbale dans cette langue ne peuvent être traitées à un niveau

* Camille Simon est doctorante au Laboratoire LACITO (UMR 7107, Université Paris 3). 
simplement syntaxique mais doivent intégrer des composantes pragmatiques et sémantiques essentielles : absence d'indexation des actants au niveau du syntagme verbal (Foucaux, 1858 : 53; Tournadre, 1996 : 70), caractère facultatif (en contexte) de tous les syntagmes nominaux d'un énoncé (Tournadre, 1996 : 68); et fluctuations dans la présence des marques casuelles : celles-ci sont omissibles pour des raisons stylistiques dans la langue littéraire, sémantico-pragmatiques dans la langue parlée (Tournadre, 2010 : 114-116). Il a également été montré que cette langue, qui suit un alignement principalement ergatif, ne connait ni voix passive ni antipassive (Tournadre, $1996: 87-98$ ), les fonctions sémantiques ou pragmatiques prises en charge par ces diathèses ${ }^{1}$ pouvant être exprimées par simple omission d'un argument ou permutation de leur ordre d'apparition dans l'énoncé.

On pourrait dès lors se demander dans quelle mesure la notion de valence est opérationnelle en tibétain. Ne serait-il pas préférable, d'abandonner l'idée selon laquelle le verbe détermine le nombre d'actants dans l'énoncé au profit d'une conception selon laquelle les verbes de cette langue ne possèdent pas de structure actancielle fondamentale et sont donc susceptibles de régir un nombre variable d'actants? Des difficultés de ce type, mais à des degrés moindres, sont fréquemment évoquées dans les descriptions typologiques de la valence verbale dans diverses langues (par exemple voir l'analyse de cette notion par Lazard, 1994), sans pour autant remettre en cause le concept même de valence comme outil de classification syntaxique des verbes.

Face à ces difficultés, une définition de la valence faisant intervenir deux niveaux a été proposée (voir par exemple Creissels, 2006) et permet de résoudre une partie des problèmes rencontrés sans remettre en cause la pertinence de la notion. On distinguera alors valence syntaxique ou transitivité, définie au niveau de l'énoncé d'une part (nombre d'actants présents dans un énoncé donné), et valence sémantique, définie au niveau du lexique d'autre part.

Dans un premier temps nous proposerons un critère pour distinguer actants et circonstants parmi les syntagmes nominaux de l'énoncé. En effet, du fait des caractéristiques morphosyntaxiques de cette langue, les critères fréquemment énumérés pour définir la valence verbale ne fonctionnent pas, ou fonctionnent mal, en tibétain.

1. C'est-à-dire, une modification de la valence verbale semblable à un changement de voix, mais non marqué sur le syntagme verbal. 
Ainsi, nous suggérons de fonder la définition de la valence en tibétain, et dans les langues dont le fonctionnement morphosyntaxique est semblable, essentiellement sur un critère sémantique : l'implication ou non par le verbe de la présence d'un Participant (dans l'énoncé ou dans le contexte).

La définition de la valence verbale rend ensuite possible l'étude des questions de réalisation morphosyntaxique des actants et de leur rôle syntaxique dans l'énoncé en permettant de mettre en évidence l'existence de deux types de diathèses en tibétain : les changements de valence de type causatif ou décausatif/autocausatif d'une part, et réciproque d'autre part. Ces changements de valence ne s'accompagnent d'aucun marquage du syntagme verbal, mais fonctionnent de façon parallèle aux voix réciproque et causative ou décausative/autocausative, tant au niveau sémantique qu'à celui du marquage des actants.

On observe également l'existence d'autres mécanismes réguliers de modification du marquage casuel des actants, accompagnés de changement sémantique. Nous décrirons plus en détail l'un d'entre eux dans la dernière partie. Ces mécanismes se distinguent des diathèses dans la mesure où ils ne peuvent être mis en parallèle avec des voix attestées par ailleurs dans d'autres langues.

Nous proposons néanmoins de considérer de la même façon les phénomènes de diathèse des autres phénomènes de variation du traitement morphosyntaxique des actants dans un énoncé, dans la mesure où ils manifestent tous deux un choix du locuteur pour conceptualiser un évènement. William Croft (2012 : 13-14) développe la notion de «conceptualisation» ou d'«interprétation» pour désigner le fait qu'un même évènement peut être décrit, dans une même langue, sous des formes diverses. Ces interprétations syntaxiques d'un évènement ont les caractéristiques suivantes (Croft, $2012: 13)^{2}$ :

a. There are multiple alternative construals of an experience available.

b. A speaker has to choose one construal or another; they are mutually exclusive.

c. No construal is the "best" or "right" one out of context".

2. «a. Plusieurs interprétations alternatives pour une expérience sont disponibles. b. Un locuteur doit choisir l'une ou l'autre de ces interprétations : elles s'excluent mutuellement.

c. Hors contexte, aucune interprétation n'est "plus juste" ou "meilleure" qu'une autre.» 
Cette notion permet en particulier d'expliquer la variabilité du traitement morphosyntaxique des actants, qu'il s'agisse ou non à proprement parler de diathèses. Ainsi, une analyse semblable des deux types de diathèses mentionnées et des autres phénomènes de variation de traitement morphosyntaxique des actants permet de montrer non seulement l'importance des facteurs sémantico-pragmatiques pour la description de la syntaxe du tibétain, mais également, à des degrés divers, pour la plupart des langues.

Les données présentées ici ont été recueillies à Lhasa, capitale traditionnelle et culturelle de la zone linguistique tibétaine, auprès d'une locutrice de 28 ans, originaire de la ville, lors d'un séjour effectué entre septembre 2009 et juillet 2010. L'aire linguistique tibétaine, qui s'étend sur un peu moins de 2,5 millions de $\mathrm{km}^{2}$ comprend de nombreux dialectes ou langues, entre lesquelles l'intercompréhension est limitée ${ }^{3}$. La langue parlée à Lhasa est une langue très proche du tibétain standard (langue de la diaspora), mais comporte néanmoins des spécificités lexicales et syntaxiques, qui transparaissent dans les énoncés présentés ici. Leur enregistrement correspondait à une entreprise de documentation des constructions syntaxiques associées aux verbes tibétains couramment employés dans la langue parlée : on a demandé à la locutrice d'illustrer les divers emplois possibles de chaque verbe, en proposant quelques énoncés dans lesquels il constituait le verbe principal.

\section{Une définition sémantique de la valence verbale}

Définir la valence d'un verbe implique d'opérer une distinction claire entre les actants centraux, dépendant directement du syntagme verbal, et les actants périphériques, ou circonstants, qui ne partagent pas cette relation avec le verbe. Gilbert Lazard (1994: 68-70) propose, comme premier critère, le fait que la présence de certains arguments, contrairement à d'autres, est syntaxiquement requise dans l'énoncé (sous forme de syntagmes nominaux ou d'indices actanciels portés par le verbe). Ce même auteur nuance cependant son propos en notant l'existence, dans des langues aussi bien connues que le français, d'actants non-requis et de circonstants qui le sont (Lazard, 1994 : 70). On constate donc que la formulation d'un tel critère s'avère, dès le départ, problématique. Les langues comme le tibétain où, comme cela a été montré (Tournadre, 1996 : 69-70), aucun syntagme nominal n'est obligatoirement présent

3. Voir par exemple à ce sujet Tournadre (2005). 
dans l'énoncé et où le verbe ne comporte aucun indice actanciel, invitent à le mettre en question, ou du moins à en reconsidérer l'importance. On peut se demander si, typologiquement, il est à retenir parmi les critères définitoires de la valence. On peut ajouter que le second critère mis en avant par Gilbert Lazard, celui des marques portées par les arguments, n'est pas non plus applicable en tibétain. En effet, les marques casuelles, qui distinguent les différents types d'arguments dans un énoncé, sont pour la plupart polysémiques dans cette langue (ergatif-instrumental; datif-locatif-directif, ablatif-ergatif, etc.). Il est donc impossible de définir un actant par son marquage morphologique. De plus, comme cela a été mentionné plus haut, elles peuvent être omises dans certaines conditions.

Il est donc nécessaire de se fonder sur d'autres critères pour définir la valence du verbe en tibétain. Nous proposons de retenir un critère d'ordre sémantique plutôt que syntaxique pour déterminer le nombre des actants d'un verbe.

Ainsi, on considérera qu'un syntagme nominal fait partie de la valence sémantique d'un verbe s'il ne peut pas être supprimé dans un énoncé hors contexte. Si son absence formelle dans un énoncé renvoie immédiatement l'interlocuteur au contexte pour reconstituer cet élément (c'est-à-dire qu'elle provoque une anaphore zéro), alors il fait partie de la valence du verbe. En l'absence de tout argument syntaxiquement requis dans l'énoncé, ce test de l'anaphore zéro est en effet le seul moyen qui permette de mettre en évidence la présence d'une entité non exprimée mais, cependant, sous-entendue par l'emploi d'un verbe particulier. Dans un second temps, le fait qu'une telle entité soit sous-entendue par le verbe indique que l'argument entretient une relation étroite avec le verbe régissant la proposition, et peut, à ce titre, être considéré comme un actant de ce verbe. Il s'agit donc d'opérer un déplacement de cette notion d'argument requis d'un niveau purement syntaxique à un niveau sémantique, ce qui, par ailleurs, est conforme avec l'idée selon laquelle la valence est une propriété lexicale du verbe. Bettina Zeisler (2007 : 402) mentionne l'utilisation de ce critère dans l'étude de la valence des verbes en Ladakhi.

Le changement ou l'absence de changement de valence sémantique est mis en évidence de la façon suivante : des énoncés, dans lesquels l'argument dont on veut tester le statut est omis, sont présentés hors contexte à un locuteur. Sa réaction face à ces énoncés indique si le syntagme nominal omis est ou non à considérer comme un actant. En effet, si le locuteur interroge spontanément son interlocuteur sur l'identité de l'élément omis, cela signifie bien que cet élément fait partie 
de la valence du verbe puisque son absence renvoie au contexte pour l'identifier. Dans ce cas, l'énoncé ne comportant qu'un seul actant est bien sémantiquement incomplet, quoique syntaxiquement correct. L'omission formelle de l'actant n'est donc pas corrélée à un changement de la valence sémantique du verbe puisqu'elle génère une anaphore.

Les exemples en (1), (2) et (3) montrent que l'emploi d'un même verbe dans un énoncé comprenant respectivement deux actants (exemples a) ou un seul (exemples b) peut ou non être accompagné d'une modification de la valence du verbe.

(1) Pas de modification de la valence

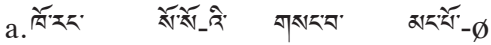
kho.rang so.so-'i gsang.ba mang.po- $\varnothing$
₹_₹
$3 \mathrm{sg}$
RÉFL-GÉN secret
beaucoup-ABS
nga-r bshad-byung/
'Il m'a dit beaucoup de ses secrets.'

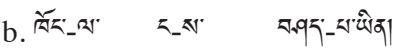
khong-la nga-s bshad-pa.yin/
3SG-DAT 1SG-ERG dire-PST1
'C'est moi qui le lui ai dit.'
‘* C'est moi qui lui ai parlé.'

Dans l'exemple (1b) l'absence de mention explicite du Patient est ainsi systématiquement interprétée comme une anaphore zéro. Le Patient fait bien partie de la grille actancielle du verbe.

Au contraire, en (2a) et (2b), on a bien un verbe régissant tantôt deux actants, tantôt un seul. En effet, l'absence d'actant marqué à l'ergatif ne génère pas d'anaphore zéro et il n'est même pas possible d'introduire un Agent dans l'exemple (2b).

(2) Modification de la valence

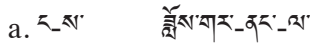

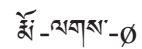

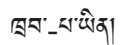
nga-s
zlos.gar-nang-la
rmo-lags- $\phi$
khrab-pa.yin/
1 SG-ERG
théâtre-intérieur-DAT
Grand.mère-H-ABS
jouer-PST 1
'Au théâtre, j’ai joué la Grand-mère.'

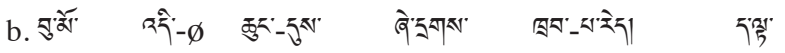
bu.mo 'di- $\phi$ chung-dus zhe.drags khrab-pa.red/ da.lta
fille DÉM-ABS petit-quand très jouer-PST3 maintenant

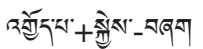
'gyod.pa+skyes-bzhag
regret+Vsup-Pst5

'Cette fille s'est beaucoup amusée quand elle était jeune.

Maintenant, elle regrette.' 
Ici, le changement de valence sémantique s'accompagne d'un glissement sémantique du verbe khrab 'jouer'. Cependant, ce n'est pas toujours le cas, comme le montrent les exemples (3a-b) avec un autre verbe :

\begin{tabular}{|c|c|c|c|}
\hline 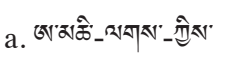 & こ_રิ & গู่- $\varnothing$ & 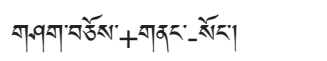 \\
\hline a.mchi-lags-kyis & $n g a-{ }^{\prime} i$ & $b u-\phi$ & gshag.bcos+gnang-song/ \\
\hline médecin-H-ERG & 1SG-GÉN & fils-ABS & opération+Vsup.H-PST4 \\
\hline
\end{tabular}

'Le médecin a opéré mon fils.'

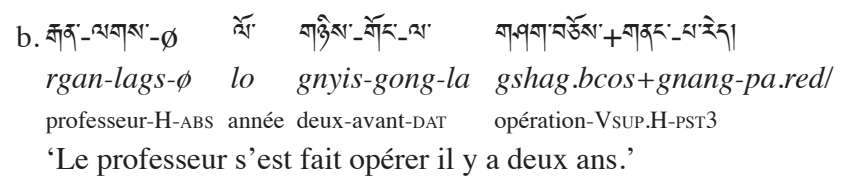

Les exemples (1) à (3) témoignent donc de l'importance de distinguer ce phénomène d'ellipse d'un actant, dans un contexte clair, d'une véritable modification de la valence sémantique du verbe. En effet, dans l'exemple (1b) l'argument absent du verbe Dort être déductible du contexte de l'énoncé, alors que ce n'est pas le cas dans les exemples (2b) et (3b). Dans ces deux derniers exemples, en effet, il s'agit d'une véritable réduction d'actants, et non d'une simple omission. Par ailleurs, l'exemple (2) témoigne d'une certaine spécialisation lexicale introduite par ce changement de valence syntaxique : les sens de l'emploi biactanciel et monoactaciel du verbe ne sont pas sémantiquement équivalents.

On constate donc, à travers ces exemples, que si les arguments ne sont pas syntaxiquement requis par le verbe, au sens de Gilbert Lazard (1994 : 69-70), il n'en va pas de même au niveau sémantique : certains arguments du verbe sont sémantiquement requis (leur absence formelle déclenche une anaphore zéro), tandis que d'autres ne le sont pas. Le nombre d'actants présents dans un énoncé (la transitivité) ne peut pas constituer un critère de définition de la valence d'un verbe en tibétain et ne peut donc être pris en compte pour examiner les phénomènes de changement de valence. En revanche, la variation de la valence sémantique constitue un critère pertinent pour définir un changement de la structure actancielle du verbe.

Cette définition de la valence verbale permet de mettre en évidence l'existence de deux types de diathèses qui correspondent - au niveau sémantique et syntaxique - à des procédés de dérivation actancielle qui, dans d'autres langues, sont marqués au niveau du syntagme verbal. 


\section{Diathèse causative ou autocausative / décausative}

Une première catégorie de changement de la valence sans corrélat formel sur le verbe concerne les cas où la réorganisation syntaxique des arguments s'accompagne d'une modification du nombre des Participants à l'évènement (donc d'une modification de la valence sémantique).

Certains verbes peuvent ainsi être employés alternativement dans une construction monoactancielle, ou dans une construction biactancielle (construction ergative ou ergative mixte ${ }^{4}$ ). Comme vu précédemment, il est nécessaire de distinguer les cas d'omission de l'Agent (marqué à l'ergatif) dans l'énoncé, produisant l'effet d'anaphore zéro comme dans l'exemple (1), des véritables cas de modification de la structure syntaxique et sémantique régie par le verbe, comme dans l'exemple ci-dessous, où la construction monoactancielle (4b) n'implique pas l'existence d'un Agent. Autrement dit, et conformément à la définition de la valence proposée dans la section 2, il est nécessaire que ce changement de transitivité s'accompagne d'un changement de la valence sémantique du verbe pour pouvoir considérer ce phénomène comme une diathèse.

(4)

\begin{tabular}{|c|c|c|c|c|}
\hline a. 2 -N & \multicolumn{2}{|c|}{ केरेश्र- $\varnothing$} & \multicolumn{2}{|c|}{ 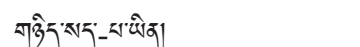 } \\
\hline$n g a-s$ & tshe. & ing- $\phi$ & \multicolumn{2}{|c|}{ gnyid.sad-pa.yin } \\
\hline 1SG-ERG & Tsérin & $-\mathrm{ABS}$ & \multicolumn{2}{|l|}{ réveiller-PST 1} \\
\hline \multicolumn{5}{|c|}{ 'J'ai réveillé Tséring.' } \\
\hline b. ${ }^{\Sigma}-\varnothing$ & देरेद & 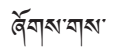 & 줄추 & 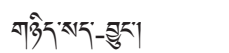 \\
\hline$n g a-\phi$ & de.ring & zhogs.gas & snga.po & gnyid.sad-byung/ \\
\hline $1 \mathrm{SG}-\mathrm{ABS}$ & aujourd'ht & matin & tôt & réveiller-PST2 \\
\hline
\end{tabular}

Il peut être difficile d'établir clairement le changement de valence dans de tels exemples. Par définition, dans le cadre d'un alignement ergatif, on n'observe pas de modification du marquage syntaxique des actants : le même argument reste marqué à l'absolutif qu'il soit actant unique de la construction monoactancielle ou second actant de la construction ergative. De plus, l'actant unique de la construction monoactancielle semble, du point de vue sémantique, devoir être assi-

4. C'est-à-dire respectivement dont le premier actant est marqué à l'ergatif et le second à l'absolutif, et dont le premier actant est à l'ergatif et le second au datif. 
milé au Patient de la construction biactancielle (à cause de l'absence de volition notamment).

De la même façon, dans les exemples (3a) et (3b), l'ambigüité de l'énoncé intransitif (3b) est mise en évidence par l'emploi d'un verbe appartenant au registre honorifique. Deux interprétations sont en effet possibles, en termes de valence :

- l'Agent est omis et reconstitué par anaphore zéro, auquel cas le choix d'un verbe honorifique s'explique par le fait que l'Agent (le médecin) est jugé digne de ce registre de langue;

- le verbe régit une construction monoactancielle simple et le choix de la version honorifique du verbe s'explique par le fait que l'actant unique (le professeur) est jugé digne de ce registre de langue.

On peut noter que la première interprétation est spontanément préférée dans un énoncé comme (5), où il est plus difficile - même si ce n'est bien sûr pas exclu - d'imaginer un contexte où l'on associerait 'fils' (dont la position sociale est inférieure à celle de ses parents ${ }^{5}$ ) à un verbe du registre honorifique. La seconde possibilité d'interprétation de cet énoncé, indiquée ci-dessous avec un point d'interrogation, est donc considérée par les locuteurs comme plus improbable, en l'absence d'un contexte particulier. Cette possibilité indique néanmoins que le verbe gshags.bcos+gnang 'opérer' (honorifique) peut bien régir deux grilles actancielles distinctes.

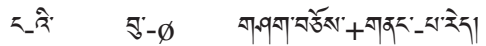

$$
\begin{aligned}
& \text { nga-'i bu- } \phi \quad \text { gshag.bcos+gnang-pa.red } \\
& \text { 1SG-GÉN fils-ABS opération-Vsup.H-Pst3 } \\
& \text { '[Il] a opéré mon fils.' Mais :? 'Mon fils s'est fait opérer.' }
\end{aligned}
$$

Par ailleurs, l'emploi des suffixes verbaux constitue également une indication sur le fait que l'énoncé ne comprenant qu'un actant marqué à l'absolutif représente véritablement un changement de valence par rapport à l'énoncé comprenant deux actants.

En (6a), l'emploi du suffixe verbal -payin, qui exprime la valeur d'égophorique contrôlable, indique que l'actant marqué à l'absolutif est bien un actant unique. En effet, ce suffixe ne peut être employé que lorsque l'actant marqué à l'absolutif en fonction d'Agent ou d'actant

5. Un contre-exemple serait le cas où le fils est un lama réincarné, auquel cas l'emploi de l'honorifique serait de mise. 
unique correspond à une première personne qui accomplit l'action intentionnellement - ce qui irait à l'encontre de sa définition comme Patient. Il est alors exclu d'insérer un Agent, comme dans l'exemple (6b).

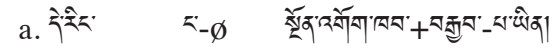
de.ring nga- $\phi$ sngon.'gog.khab+brgyab-pa.yin/
aujourd'hui 1sG-ABS vaccin+VsuP-PST1
'Aujourd'hui, je me suis fait vacciner.'

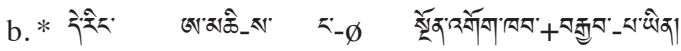
* de.ring a.mchi-s nga-ф sngon.'gog.khab+brgyab-pa.yin/
aujourd'hui médecin-ERG 1sG-ABS vaccin+Vsup-PST1
Lit. '* Aujourd'hui, je me suis fait vacciner par le médecin.'

Enfin, pour mentionner de façon exhaustive l'ensemble des possibilités, on peut encore donner le cas où le seul actant apparaissant dans l'énoncé est marqué au datif (7a) ou à l'ergatif (7b). En (7a) le fait que l'actant apparaisse au datif implique qu'il s'agit d'un Patient grammatical. Dans ce cas, lorsque l'Agent est omis, son identité est nécessairement indiquée par le contexte (le marquage du Patient grammatical au datif plutôt qu'à l'absolutif correspond ici à une emphase sur son identité - voir également à ce sujet la section 5). L'exemple (7b) est potentiellement ambigu dans la mesure où l'actant marqué à l'ergatif peut être l'Agent (donc un médecin) - et le Patient serait sousentendu - mais il peut également être analysé comme l'actant unique d'un verbe lorsque celui-ci est mis en contraste avec un autre actant (emphase contrastive, qui explique l'emploi de l'ergatif dans un énoncé monoactanciel, voir Tournadre, $1996: 300$ ).
a. रेरें
ขั้วร:-2ง

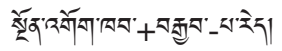
de.ring
mo.rang-la
sngon.'gogkhab+brgyab-pa.red/
aujourd'hui
3SG-DAT
vaccin+Vsup-PST3
'Aujourd'hui, il a été vacciné.' / 'Aujourd'hui, on/il/elle l'a vacciné.'

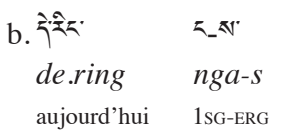

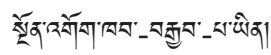
sngon.'gog.khab+brgyab-pa.yin/
'Aujourd'hui, je me suis fait vacciner.' Ou bien 'Aujourd'hui, je l'ai vacciné.'

Ainsi pour ces verbes, un énoncé ne comprenant qu'un seul actant marqué à l'absolutif peut être compris comme une véritable construction monoactancielle (sans Agent) ou bien, alternativement, comme 
une construction ergative où l'Agent serait sous-entendu. Par contre, un énoncé ne comprenant qu'un seul actant marqué au datif implique nécessairement, au niveau sémantique, la présence d'un Agent sousentendu. Dans le premier cas, on a donc bien une modification de la valence du verbe, qui peut être mise en parallèle avec la diathèse causative ou décausative / autocausative (l'orientation étant difficile à déterminer du fait de l'absence de marque de dérivation actancielle sur le verbe). Selon Denis Creissels (2006 : 31), les formes autocausatives peuvent être définies de la façon suivante :

[E]lles suggèrent que le déclenchement du processus ne met en jeu aucune force extérieure identifiable (La porte s'ouvre, Le tissu s'use), ou bien elles minimisent l'intervention d'une force extérieure en insistant sur une prédisposition particulière du référent sujet à subir le procès (Ce tissu se lave facilement).

Le décausatif est ici distingué de l'autocausatif : l'actant unique d'un énoncé autocausatif est susceptible de contrôler l'évènement, contrairement à celui d'un énoncé décausatif.

Notons au passage qu'il est exclu de rapprocher une telle alternance d'un mécanisme de passivisation, en adoptant la définition du passif du même auteur :

Le passif canonique est un mécanisme qui, opérant sur un verbe transitif, produit une forme intransitive dérivée dont le sujet reçoit exactement le même rôle sémantique que l'objet de la construction transitive. L'argument représenté par le sujet de la construction transitive peut devenir un oblique ou être complètement occulté mais sa participation même à l'évènement reste impliquée par la construction passive. (Creissels, 2006 : 9)

On a vu plus haut, dans la section 1, que la modification du nombre d'arguments du verbe en tibétain ne pouvait être définie qu'au niveau sémantique : implication ou non de la présence d'un Participant mise en évidence principalement par l'anaphore zéro ainsi que de façon plus secondaire, par les suffixes verbaux et l'emploi de l'honorifique. La définition du passif telle qu'elle est donnée par cet auteur entre donc en contradiction avec les critères adoptés ici pour définir la valence verbale en tibétain.

D'autre part, la possibilité, comme en (6a), d'avoir un suffixe verbal indiquant que l'actant unique contrôle le déroulement de l'évènement implique qu'il y ait une réorganisation du rapport entre rôles sémantiques et syntaxiques, et non une simple permutation. En (6a) l'actant 
unique ne correspond pas seulement au Patient sémantique de l'évènement mais cumule certaines caractéristiques sémantiques à la fois de l'Agent et du Patient.

Ainsi, selon les définitions du décausatif et de l'autocausatif, l'exemple (7a) qui comporte un suffixe verbal indiquant que l'actant unique contrôle le déroulement du procès, se rapprocherait d'une forme autocausative. À l'inverse, l'exemple (4b), dans lequel le suffixe verbal -byung indique l'absence de contrôle de l'évènement par l'actant unique, peut être mis en parallèle avec l'exemple suivant, illustrant le décausatif :

(8) Tswana (Creissels, $2006: 37)$
a. Ngwana
o thub-il-e
mae.
CL1.enfant s3.CL1 casser-PARF-FIN
CL6.œuf

'L'enfant a cassé les œufs.'

$\begin{array}{lll}\text { b. Mae } & a & \text { thub-eg-il-e. } \\ \text { CL6.æuf } & \text { s3.CL6 } & \text { casser-DÉCAUS-PARF-FiN }\end{array}$

'Les œufs se sont cassés.'

Comme on l'a vu, il est également possible de comparer cette alternance de construction syntaxique à la dérivation causative. En effet, si l'on considère que la forme de base est l'énoncé intransitif, on observe un fonctionnement parallèle à la dérivation causative définie par l'ajout d'un Agent causateur à l'évènement. Ainsi il est possible de comparer les exemples en (7) aux exemples en (9).

(9) Turc (Ersen-Rasch, $2001:$ 193)
a. [Ben- $\varnothing]$ bekle-di-m
[1SG-Nom] attendre-PST-1sG
'J'ai attendu.'
b. [Sen- $\varnothing]$ ben-i bekle-t-t-in.
[2SG-NOM] 1SG-ACC attendre-CAUS-PST-2SG
'Tu m'as fait attendre.'

On peut donc établir un parallèle entre l'alternance des constructions syntaxiques possibles avec un verbe donné en tibétain et des changements de voix. Il faut cependant rappeler que, pour cette alternance de valence, il est souvent loin d'être évident d'évaluer si, au niveau sémantique, l'actant externe (Agent ou causateur) est ou non présent dans la construction monoactancielle. $\mathrm{Si}$, dans le cas de certains verbes présentés ci-dessus, on peut dire qu'on se trouve véritablement en présence d'un changement de valence, dans d'autres cas, il est plus 
difficile d'écarter la possibilité d'une simple anaphore zéro ou d'un Agent restant indéfini. Cette ambigüité rend donc également compliquée l'évaluation de la fréquence et de la productivité de cette alternance syntaxique.

Quoi qu'il en soit, l'existence de ce type d'alternance plaide en faveur de la distinction entre valence sémantique et transitivité (au niveau de l'énoncé) puisque seule cette distinction permet de mettre en évidence l'existence d'une catégorie de verbes capables de régir alternativement un ou deux actants en tibétain, en permettant de faire ressortir une catégorie syntaxique particulière de verbes dans cette langue, verbes dont le comportement s'apparente aux verbes dits «labiles» ou «ambitransitifs » selon d'autres terminologies.

\section{Diathèse réciproque}

Deux types d'alternance de l'alignement des actants régis par le verbe impliquent la marque de l'associatif et peuvent, comme nous allons le montrer, être considérés comme des diathèses réciproques. Tout d'abord, on observe une alternance entre une construction ergative mixte (biactancielle, ergatif-datif), une construction monoactancielle (dont l'actant unique est donc marqué à l'absolutif), et une construction biactancielle «associative», dans laquelle le premier actant est marqué à l'ergatif et le second à l'associatif. Les exemples ci-dessous illustrent les deux premiers types de constructions mentionnées :
'Cette laine s'est emmêlée.'

Dans l'exemple (10b), Tashi et Tséring (bkra.shis dang tshe. ring- $\emptyset$ ) forment un seul constituant syntaxique, là où on en a deux en (10a). On a bien ici un changement de diathèse dans la mesure où 
un même verbe régit tantôt une construction avec un actant unique marqué à l'absolutif (10b), tantôt une construction biactancielle où le premier actant est marqué à l'ergatif et le second actant au datif (10a). L'exemple (10c) montre par ailleurs que la construction monoactancielle n'implique pas nécessairement la présence d'un actant unique pluriel ou collectif.

Le sémantisme lié à cette alternance de marquage syntaxique nous invite par ailleurs à comparer le fonctionnement de ces énoncés à celui de la voix réciproque, et plus généralement, aux voix moyennes, dont le réciproque constitue une sous-catégorie.

En effet, Denis Creissels (2006 : 9-10) note :

Les formations morphologiques traditionnellement désignées comme voix moyennes encodent typiquement des opérations sur la valence qui, appliquées à des verbes transitifs, aboutissent à des constructions intransitives dont le sujet reçoit un rôle [sémantique] qui ne s'identifie pas purement et simplement à l'un de ceux que le verbe assigne à son sujet et à son objet, mais qui retient de manière variable des traits caractéristiques de l'un ou l'autre de ces deux rôles. On peut parler de remodelage des rôles sémantiques, par contraste avec le simple réarrangement syntaxique des rôles sémantiques qui caractérise le passif.

Et il poursuit (2006 : 34) :

Une utilisation cohérente de la terminologie devrait conduire à reconnaitre comme relevant au moins partiellement de la notion de voix moyenne toute marque morphologique dont la présence dans une forme verbale caractérise cette forme comme apte à exprimer une variété de significations qui, dans le détail, peuvent relever des types suivant: réfléchi (de l'objet ou du datif), réciproque, autocausatif, décausatif, autobénéfactif).

Dans le cas de l'alternance observée pour le tibétain, on a bien, du point de vue syntaxique, une modification de l'alignement correspondant à une opération sur la valence de type réciproque. On peut en effet comparer la modification de l'organisation des arguments dans les énoncés en (10) et exemples en (11).

(11) Tswana (Creissels, $2006: 36$ )
a. Kitso
rat-a
Lorato thata
CL1Kitso s3.cL1 aimer-FIN CL1Lorato beaucoup

'Kitso aime beaucoup Lorato.' 
b. Kitso le Lorato ba rat-an-a thata
CL1Kitso avec cL1Lorato s3.cL2
'Kitso et Lorato s'aiment beaucoup.'

$\begin{array}{cllll}\text { c. Kisto } & o & \text { rat-an-a } & \text { le Lorato } \\ \text { CL1Kitso } & \text { s3.cL1 } & \text { aimer-RÉc-FIN } & \text { avec cL1Lorato }\end{array}$

'Kitso a une relation amoureuse avec Lorato.'

(Lit. 'Kitso s'aime avec Lorato.')

Les exemples (10a) et (11a) sont comparables dans la mesure où ils comportent chacun deux actants assurant deux fonctions syntaxiques distinctes. En (10b) et (10c), de même qu'en (11b), on constate bien une réorganisation de la correspondance entre rôles sémantiques et rôles grammaticaux qui ne se résume pas à une simple permutation. En effet, l'actant unique de ces trois exemples cumule les rôles sémantiques des deux actants présents respectivement dans les énoncés en (10a) et (11a) : il est à la fois Agent et Patient sémantique.

On trouve de plus en tibétain comme en tswana, une construction biactancielle où le premier actant est à l'ergatif et le second à l'associatif, illustrée en (11c) pour le tswana et en (12) pour le tibétain.

\begin{tabular}{|c|c|c|}
\hline 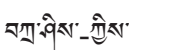 & 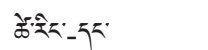 & 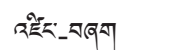 \\
\hline bkra.shis-kyis & tshe.ring-dang & 'dzing-bzhag \\
\hline Tashis-ERG & Tséring-ASS & se.battre-PST5 \\
\hline
\end{tabular}

On observe, par ailleurs, que la construction ergative, dans laquelle le second actant est marqué à l'absolutif, n'est pas possible :

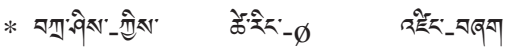

$$
\begin{aligned}
& \text { * bkra.shis-kyis tshe.ring- } \phi \quad \text { 'dzing-bzhag } \\
& \text { Tashi-ERG Tséring-ABS se.battre-PST5 }
\end{aligned}
$$

Au niveau sémantique, la construction monoactancielle et la construction associative d'une part, et la construction ergative mixte (un actant à l'ergatif et le second au datif) d'autre part n'ont pas les mêmes implications. En effet, les deux premières impliquent une réciprocité de l'évènement. Ainsi le fait que le second Participant soit inanimé est problématique dans les énoncés (14b) et (14c), puisqu'un élément inanimé, par définition, n'est pas susceptible d'éprouver de l'affection.

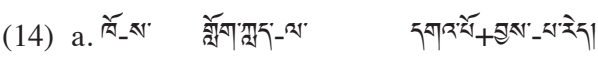

$$
\begin{aligned}
& \text { kho-s glog.klad-la dga'.po+byas-pa.red/ } \\
& \text { 3SG-ERG ordinateur-DAT appréciation+VsuP-PST3 } \\
& \text { 'Il a apprécié l'ordinateur.' }
\end{aligned}
$$



b. ?

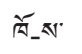

Lit. 'Il s'est apprécié avec l'ordinateur.'
बेंखर

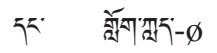

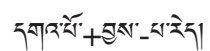
? kho.rang $3 \mathrm{sG}$
dang glog.klad- $\phi$
et ordinateur-ABS
dga'.po+byas-pa.red/
apprécier-PST3

Lit. 'Lui et l'ordinateur se sont appréciés.'

De la même façon dans les exemples en (15), l'absence de réciprocité de l'évènement, clairement établie par le contexte, rend sémantiquement impossible l'emploi des constructions associative et monoactancielle :

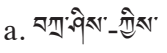
bkra.shis-kyis

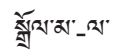

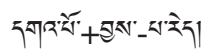
Tashi-ERG
थेनकरें
sgrol.ma-la
dga'.po+byas-pa.red/ yin.na' $i$

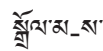
Drölma-DAT
appréciation+Vsup-PST3
mais
sgrol.ma-s

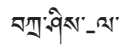

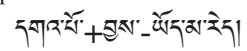
s bkra.shis-la
dga'.po+byas-yod.ma.red/

'Tashi a apprécié Drölma. Mais Drölma n’a pas apprécié Tashi.'

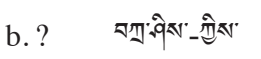

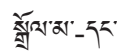

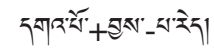
? bkra.shis-kyis
sgrol.ma-dang
dga'.po+byas-pa.red/
Tashi-ERG
Drölma-Ass

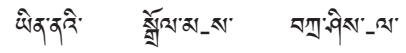
appréciation+Vsup-PST3
yin.na'i sgrol.ma-s bkra.shis-la

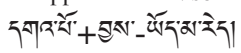
mais
Drölma-ERG Tashi-DAT
dga'.po+byas-yod.ma.red/

Lit. 'Tashi s'est apprécié avec Drölma. Mais Drölma n'a pas apprécié Tashi.'

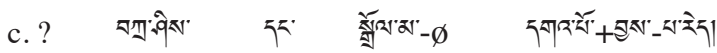
? bkra.shis dang sgrol.ma- $\phi$ dga'.po+byas-pa.red/
Tashi et Drölma-ABS appréciation+Vsup-Psт3

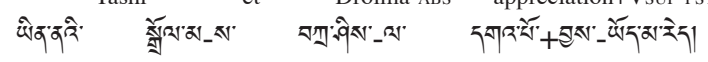

yin.na'i sgrol.ma-s bkra.shis-la dga'.po+byas-yod.ma.red/

mais Drölma-ERG Tashi-DAT appréciation+VsuP-PST3.NÉG

Lit. 'Tashi et Drölma se sont appréciés. Mais Drölma n'a pas apprécié Tashi.'

Ainsi la construction monoactancielle avec ce groupe de verbes, lorsque l'actant unique est pluriel ou collectif, implique la réciprocité, c'est-à-dire que cet actant unique représente en même temps les rôles sémantiques représentés par les deux actants marqués à l'ergatif et à l'associatif ou au datif dans les constructions biactancielles. La 
construction dans laquelle le second actant est marqué à l'associatif implique également une réciprocité et donc une répartition des caractéristiques sémantiques des Participants sur les deux actants du verbe. Au contraire, celle où le second actant est marqué au datif n'implique pas une telle réciprocité et correspond, dans les langues où il existe une voix réciproque, à la construction de base. La sémantique confirme donc que cette alternance de constructions syntaxiques est bien à mettre en parallèle avec les mécanismes de voix réciproque.

On rencontre en tibétain un second type d'alternance de constructions syntaxiques impliquant la marque de l'associatif. Cette alternance fonctionne de façon parallèle à celle décrite ci-dessus mais avec des énoncés comportant un actant supplémentaire :

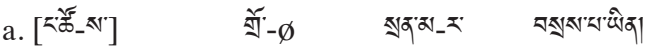 [nga-tsho-s] gro- $\phi \quad$ sran.ma-r bsras-pa.yin/ [1SG-PL-ERG] blé-ABS fève-DAT mélanger-PST1 'Nous avons mélangé du blé aux fèves (intentionnellement).'

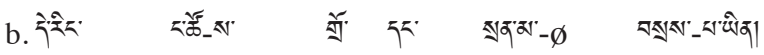 de.ring nga-tsho-s gro dang sran.ma- $\phi$ bsras-pa.yin/ aujourd'hui 1SG-PL-ERG blé et fève-ABS mélanger-PST1
'Aujourd'hui nous avons mélangé du blé et des fèves.'

\begin{tabular}{|c|c|c|c|}
\hline 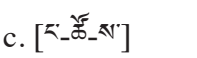 & त्रें- & $\sqrt{9} \alpha^{\prime}-7^{\circ}$ & 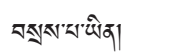 \\
\hline [nga-tsho-s] & gro- $\phi$ & sran.ma-dang & bsras-pa.yin/ \\
\hline [1SG-PL-ERG] & blé-ABS & fève-Ass & mélanger-PST1 \\
\hline
\end{tabular}

On retrouve en effet, comme dans les exemples présentés en (10) et (12), le même type de modification de l'organisation syntaxique des Participants : d'une construction triactancielle, avec un Patient grammatical marqué à l'absolutif et un destinataire marqué au datif (16a), on passe à une structure biactancielle avec Patient unique collectif marqué à l'absolutif en (16b), et enfin on a en (16c) une structure qui comprend un Patient grammatical marqué à l'absolutif, et un troisième actant (un co-Patient) marqué à l'associatif. Cependant, à la différence des constructions précédemment décrites, le rôle sémantique de l'Agent n'est ici pas concerné par les variations d'alignement : la réorganisation des rôles sémantiques et syntaxiques se fait au niveau du Patient grammatical, représenté par un seul actant en (16c) et par deux actants en (16a) et (16b).

On peut donc comparer les alternances de marquage pour ces deux groupes de verbes sous la forme du tableau suivant : 


\begin{tabular}{|c|c|c|c|c|}
\hline \multicolumn{2}{|c|}{ Groupe 1 } & \multicolumn{3}{c|}{ Groupe 2 } \\
\hline Actant 1 & Actant 2 & Actant 1 & Actant 2 & Actant 3 \\
\hline \multicolumn{2}{|c|}{ ABS } & ERG & \multicolumn{2}{c|}{ ABS } \\
\hline ERG & AsS & ERG & ABS & Ass \\
\hline ERG & DAT & ERG & ABS & DAT \\
\hline
\end{tabular}

Tableau 1. - Alternance de marquage casuel des actants dans les deux diathèses réciproques.

Enfin, en ce qui concerne la productivité de cette alternance, nous n'avons pu noter aucun verbe où serait possible un alignement syntaxique dans lequel un actant serait marqué à l'associatif, mais où les deux autres structures syntaxiques seraient impossibles. Ces deux types d'alternance exprimant la réciprocité semblent donc très réguliers.

\section{D'autres cas de variation du marquage casuel}

Il existe néanmoins d'autres cas d'alternance régulière de constructions syntaxiques en tibétain, corrélées à une modification sémantique régulière mais qui ne fonctionnent pas de façon parallèle à un mécanisme de voix. Cependant, comme les deux diathèses présentées précédemment, ces alternances manifestent un choix du locuteur pour la conception syntaxique de l'énoncé, en fonction de critères sémantiques et pragmatiques. Par ailleurs, elles permettent également de définir des sous-groupes syntaxiques parmi les verbes et donc d'aboutir à une classification plus fine dans le cadre de la description de la valence des verbes. Celles de ces alternances qui mettent en jeu la marque de l'ergatif ont fait l'objet de descriptions précises (en particulier Tournadre, 1996). Nous nous contenterons ici de décrire des cas d'alternance mettant en jeu la marque du datif qui alterne avec celle de l'absolutif dans différents types de constructions. Les effets sémantiques de cette variation de la construction syntaxique se déclinent en deux catégories :

- actant unique (dans le cadre d'une construction monoactancielle) ou Patient (dans le cadre d'une construction biactancielle) partiellement affecté par l'évènement lorsqu'il reçoit la marque du datif;

- emphase contrastive sur l'actant marqué au datif.

Les exemples (17a) et (17b) illustrent la première catégorie d'effets sémantiques associés à la présence de la marque du datif : le contexte 
donné en (17a') pour (17a) est plus difficilement compatible avec (17b) et le contexte donné en (17b') pour (17b) est plus difficilement compatible avec (17a) :

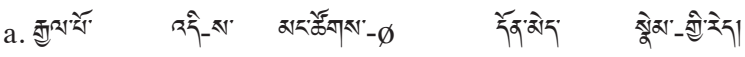

$$
\begin{aligned}
& \text { rgyal.po 'di-s mang.tshogs- } \phi \text { don.med snem-gyi.red/ } \\
& \text { roi DÉM-ERG peuple-ABS sans.raison opprimer-Fut }
\end{aligned}
$$

'Ce roi opprime le peuple sans raison.'

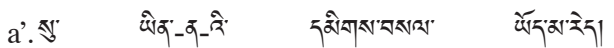

\begin{tabular}{|c|c|c|c|}
\hline 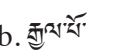 & 'दि_-N' & 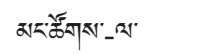 & रॅन बेन \\
\hline rgyal.po & 'di-s & mang.tshogs-la & don.med \\
\hline
\end{tabular}

$$
\begin{aligned}
& \text { su yin-na-'i dmigs.bsal yod.ma.red/ } \\
& \text { qui être-si-même spécial NÉG.exister }
\end{aligned}
$$

'Qui que l'on soit, il n'y a pas de traitement de faveur.'

'Ce roi opprime le peuple sans raison.'

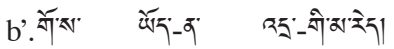

$$
\begin{aligned}
& \text { go.sa yod-na 'dra-gi.ma.red/ } \\
& \text { place exister-si être.semblable-Fut.NEG }
\end{aligned}
$$

'[Mais] si on a une [bonne] situation, alors c'est différent.'

Cette valeur sémantique associée à la présence de la marque du datif est d'ailleurs mentionnée par Dorzhi Gdongdrug Snyemsblo (1987 : 11-12), même s'il ne donne pas d'exemple illustrant spécifiquement la possibilité d'employer ces deux constructions avec un même verbe :

Entre un verbe exprimant une action affectant entièrement son Patient (bya.yul) et ce Patient, on ne place pas de marque de datif. En revanche, pour une action qui n'affecte pas son Patient ou dont seule une partie (cha.shas) du Patient est affectée, il faut une marque de datif entre le verbe et le Patient ${ }^{6}$.

En cas d'emphase sur l'identité du Patient, le marquage au datif est également préféré comme le montrent les exemples (18a) et (18b) :

6. Texte original :

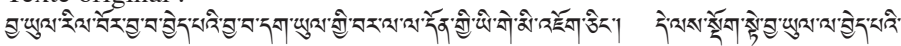

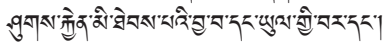

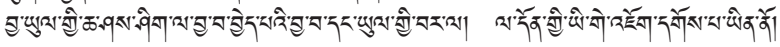




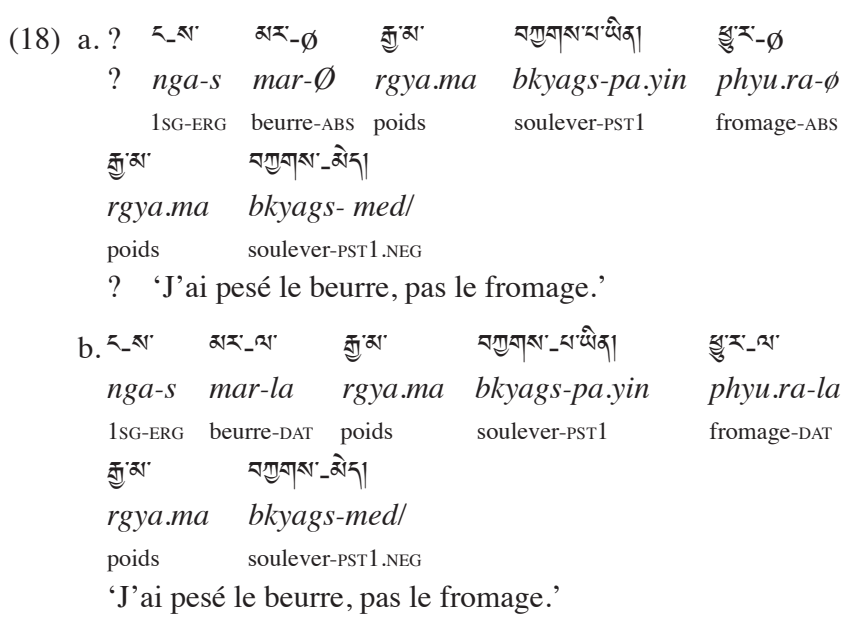

Cette valeur sémantique est mentionnée par Nicolas Tournadre dans Skalbzang 'Gyurmed (1992:26) : «L'utilisation de l'oblique à la place de l'absolutif crée une focalisation sur l'objet.»

Comme les diathèses, cette alternance constitue une modification de la relation entre le rôle sémantique des Participants à l'évènement et leur traitement morphosyntaxique. En effet on a, dans les deux cas, deux actants représentant les deux mêmes rôles sémantiques d'Agent et de Patient mais avec un traitement syntaxique différent. En (18a), le rôle grammatical de Patient est attribué au second actant tandis qu'en (18b), ce second actant est syntaxiquement traité comme un destinataire marqué au datif.

Notons par ailleurs que la même différence s'observe avec un actant unique, comme illustré en (19). Ces exemples montrent que la construction syntaxique dans laquelle l'actant unique est marqué au datif n'est pas possible si celui-ci a été entièrement affecté par l'évènement, tandis que les exemples (19c) et (19d) montrent qu'elle est préférée lorsque le Patient sémantique n'est que partiellement ou légèrement affecté. 


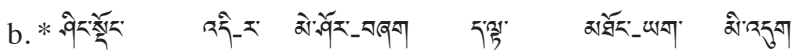
* shing.sdong'di-r me.shor-bzhag/da.lta mthong-yag mi.'dug arbre DÉM-DAT prendre.feu-PST5 maintenant voir-NTEUR NÉG-exister * 'Cet arbre a pris feu. Maintenant, il n'y a plus rien à voir [de l'arbre].'

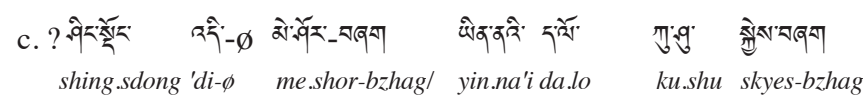
arbre DÉM-ABS prendre.feu-PST5 mais cette.année pomme pousser-PST5 '? Cet arbre a pris feu. Mais cette année, des pommes ont poussé.'

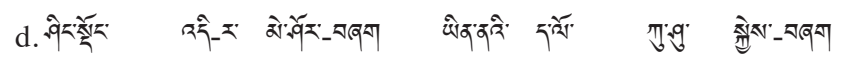
shing.sdong 'di-r me.shor-bzhag/ yin.na'i da.lo ku.shu skyes-bzhag arbre DÉM-DAT prendre.feu-PST5 mais cette.année pomme pousser-PST5 'Cet arbre a pris feu. Mais cette année, des pommes ont poussé.'

On observe donc une corrélation régulière entre une modification de l'alignement syntaxique des actants et une variation sémantique. L'alternance n'étant pas marquée dans le syntagme verbal, il est là encore difficile d'établir une orientation à cette alternance de marquage morphosyntaxique.

Néanmoins on peut, selon Gilbert Lazard (1994 : 256-257), parler d'une construction sémantiquement ou pragmatiquement marquée, qui serait donc dérivée d'une construction de base pour arriver à cette notion d'orientation. La construction de base serait donc, selon cette logique, celle où le second actant est marqué à l'absolutif.

Du point de vue formel, le mécanisme de dérivation actancielle qui semble le plus proche de cette alternance de marquage est l'antipassif. En effet, selon la définition donnée par Denis Creissels (2006 : 12) :

À l'antipassif, l'argument représenté par l'objet de la construction transitive est ou bien totalement absent, ou bien «récupéré» sous forme d'oblique.

Dans le cas du tibétain, on peut donc se demander si l'on est face à une opération sur la valence de type «antipassif» où l'actant représentant le rôle sémantique de Patient serait présent sous la forme syntaxique de cible. La comparaison des exemples suivants montre le parallèle - au niveau syntaxique - entre voix antipassive et ce type d'opération sur la valence verbale en tibétain. Ainsi, en (20) comme en (21), le second actant passe d'une absence de marque morphosyntaxique (marque de l'absolutif) dans les exemples (20a) et (21a) à un marquage comme un actant oblique en (20b) et (21b) : 
(20) K'ichee' (Creissels, 2006 : 94)
a. $X-o j-u-t i^{\prime}$
$r i$
kumatz
ACP-o1PL-s3sG-piquer DEF serpent

'Le serpent nous a piqués.'
b. Aree ri kumatz $x$ - $\emptyset$-ti'-ow q-eech
FOC DEF serpent ACP-s3sG-piquer-ANTIPASS 1PL-pour
'C'est le serpent qui nous a piqués.'

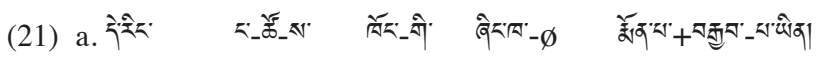

de.ring nga-tsho-s khong-gi zhing.kha-ф rmon.pa+brgyab-pa.yin/

aujourd'hui 1-PL-ERG 3.H-GÉn champ-ABS labour+Vsup-PST1

'Aujourd'hui, nous avons labouré son champ.'

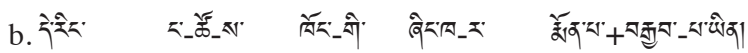

de.ring nga-tsho-s khong-gi zhing.kha-r rmon.pa+brgyab-pa.yin/

aujourd'hui 1-PL-ERG 3.H-GÉN champ-DAT labour+Vsup-PsT1

'Aujourd'hui, nous avons labouré dans son champ.'

Cependant, comme le montre l'exemple (22), le passage à la voix antipassive d'une construction biactancielle aboutit en principe à une construction monoactancielle et donc pour les constructions biactancielles ergatives, à un marquage à l'absolutif de l'Agent sémantique qui devient, au niveau syntaxique, l'actant unique de la construction.

(22) Yup'ik (Creissels, $2006: 87$ )
a. arna-m allg-aa
femme-ERG déchirer-DECL.s3SG.03SG
'La femme déchire la chemise.'
b. arna-q allg-i-uq 'lumarra-mek
femme déchirer-ANTIPASS- DECL.S3SG chemise-ABL
'La femme déchire la chemise.'

\section{'lumarraq \\ chemise}

Les exemples présentés ici montrent que ce n'est pas le cas en tibétain : l'Agent conserve sa marque d'ergatif. Il n'y a donc pas, au niveau syntaxique, de véritable parallélisme entre cette alternance de marquage en tibétain et les opérations de type antipassif.

Ainsi, plutôt que de chercher à assimiler cette alternance de marquage à un mécanisme de voix, il parait plus intéressant de suivre la proposition de Gilbert Lazard (1994 : 235-236) qui oppose plusieurs constructions syntaxiques possibles pour un même verbe, corrélées à des valeurs sémantiques propres, mais sans chercher à les rattacher au fonctionnement d'un mécanisme de changement de voix : 
L'opposition entre les deux constructions est généralement en corrélation avec la nature du procès, le degré d'affectation de l'objet, l'aspect, parfois la négation. La construction indirecte exprime souvent une action tentée, mais dont il n'est pas sûr qu'elle atteigne son objet ou qu'elle l'atteigne complètement. [...] S'opposent d'un côté la construction biactancielle majeure, qui exprime (ou suggère) un procès complet, perfectif, affectant entièrement son objet, de l'autre, la construction indirecte, qui indique un procès incomplet, seulement tenté ou en cours, qui n'atteint pas ou pas entièrement son objet, voire un procès inexistant.

Ces alternances observées en tibétain doivent donc être rapprochées de ce phénomène, généralement décrit sous le terme de «marquage différentiel » d'un actant.

\section{Conclusions}

Ainsi, trois types d'alternances syntaxiques en tibétain de Lhasa ont été décrites. Les deux premières s'apparentent à des phénomènes de diathèse (diathèse causative ou autocausative/décausative d'une part, et diathèse réciproque d'autre part) dans la mesure où elles fonctionnent de façon semblable à deux types de voix couramment observées dans les langues du monde. La troisième alternance décrite, au contraire, ne correspond à aucun phénomène de voix connu par ailleurs mais relève du même principe selon lequel des facteurs sémantiques et pragmatiques jouent un rôle dans le choix opéré par le locuteur pour le traitement morphosyntaxique des actants dans l'énoncé. Un phénomène similaire mettant en jeu la marque du datif est cependant décrit pour de nombreuses langues sous l'expression, en particulier, de «marquage différentiel de l'objet». Ces trois phénomènes peuvent néanmoins être analysés sur un même plan, considérant qu'ils témoignent tous trois de l'importance du facteur sémantique pour la description de la valence, tandis que le niveau syntaxique n'en est que le moyen d'expression. D'une manière générale, cette observation concorde d'ailleurs avec l'existence, en tibétain, d'une pluralité de constructions syntaxiques correspondant à des classes sémantiques de verbes relativement claires (Tournadre \& Sangda Dorje, 2003). Ainsi, plutôt que de vouloir traiter la valence verbale et les changements de valence comme des mécanismes syntaxiques automatiques, il parait préférable de chercher à décrire d'abord les valeurs sémantiques et pragmatiques véhiculées par les marques portées par les actants qui, à leur tour, motivent la structure syntaxique observée dans les énoncés. 


\section{ABRÉVIATIONS}

première personne

2 deuxième personne

3 troisième personne

ABL ablatif

ABS absolutif

ACC accusatif

ACP accompli

ANTIPASS antipassif

ASS associatif

CL1 classe nominale 1

CL2 classe nominale 2

CL6 classe nominale 6

CAUS causatif

DAT datif-locatif-directif

DÉCAUS décausatif

DÉM démonstratif

DECL déclaratif

DÉF défini

ERG ergatif

FIN finale

FOC focalisation

GÉN génitif

h humilifique

$\mathrm{H}$ honorifique

NÉG négatif

NOM nominatif

NTEUR nominalisateur

o objet

PARF parfait

PST1 accompli égophorique

PST2 accompli égoréceptif

PST3 accompli factuel

PST4 accompli constatif

PST5 parfait inférentiel

PL pluriel

RÉC réciproque

RÉFL pronom réfléchi

s sujet

SG singulier

VsuP verbe support 


\section{RÉFÉRENCES BIBLIOGRAPHIQUES}

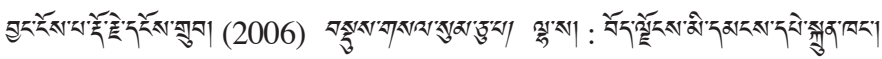

[Byangngospa Rdorje Dngosgrub (2006) : Bsdusgsal sumcupa. Lhasa, Bodljongs midmangs dpeskrunkhang.]

CREISSELS D. (2006) : Syntaxe générale, une introduction typologique 2 : La phrase, Paris, Lavoisier.

Croft W. (2012) : Verbs, Aspect and Causal Structure, Oxford, Oxford University Press.

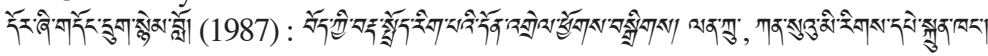

[Dorzhi Gdongdrug Snyemsblo (1987): Bod-kyi brdasprod rigpa-'i don'grel phyogsbsrigs, lankru (Lanzhou), kansu'u mirigs dpeskrunkhang.]

ERSEN-Rasch M. (2001) : Türkische Grammatik für Anfänger und Fortgeschrittene, Ismaning, Max Hueber Verlag.

Foucaux P. E. (1858) : Grammaire de la langue tibétaine, Paris, Librairie orientale de Benjamin Duprat.

LaZArd G. (1994) : L'actance, Paris, Presses universitaires de France.

SkAlbZANG 'Gyurmed (1992) : Le Clair Miroir. Enseignement de la grammaire tibétaine, traduit, adapté et commenté par Heather Stoddard et Nicolas Tournadre, La Rochette, éditions Prajna.

TOURNADRE N. (1996) : L'ergativité en tibétain : approche morphosyntaxique de la langue parlée, Louvain, Peeters.

Tournadre N. (2005) : «L'aire linguistique tibétaine et ses divers dialectes », Lalies, 25, 7-58.

Tournadre N. (2010) : «The Classical Tibetan cases and their transcategoriality, From sacred grammar to modern linguistics », Himalayan Linguistics, 9/2, 87-125.

Tournadre N. et SAngda Dorje (2003) : Manuel de tibétain standard, langue et civilisation, Paris, L'Asiathèque.

ZeISLER B. (2007) : «Case patterns and pattern variation in Ladakhi», dans $R$. Bielmeyer et F. Haller (dir.), Linguistics of the himalayas and beyond, Berlin, Mouton de Gruyter. 